\title{
Erratum to: Computational Information Geometry
}

\author{
Frank Nielsen, Frank Critchley and Christopher T.J. Dodson
}

\section{Erratum to:}

\section{F. Nielsen et al. (eds.), Computational Information Geometry, Signals and Communication Technology, DOI 10.1007/978-3-319-47058-0}

The book was inadvertently published with abstracts only in the online version and also without processing the color figures in the print version. Abstracts are added in erratum chapter and the book has been updated with the color figures.

\section{Chapter 1}

We give a personal view of what Information Geometry is, and what it is becoming, by exploring a number of key topics: dual affine families, boundaries, divergences, tensorial structures, and dimensionality. For each, we start with a graphical illustrative example (Sect. 1.1), give an overview of the relevant theory and key references (Sect. 1.2), and finish with a number of applications of the theory (Sect. 1.3).

The updated original online version for this book can be found at 10.1007/978-3-319-47058-0

\author{
F. Nielsen $(\square)$ \\ Laboratoire d'Informatique (LIX), Ecole Polytechnique, Palaiseau, France \\ e-mail: nielsen@lix.polytechnique.fr \\ F. Nielsen \\ Sony Computer Science Laboratories, Inc., Tokyo, Japan \\ F. Critchley \\ School of Mathematics and Statistics, The Open University, Milton Keynes, UK \\ e-mail: f.critchley@open.ac.uk \\ C.T.J. Dodson \\ Department of Mathematics, University of Manchester, Manchester, UK \\ e-mail: ctdodson@manchester.ac.uk


We treat 'Information Geometry' as an evolutionary term, deliberately not attempting a comprehensive definition. Rather, we illustrate how both the geometries used and application areas are rapidly developing.

\section{Chapter 2}

In statistical practice model building, sensitivity and uncertainty are major concerns of the analyst. This paper looks at these issues from an information geometric point of view. Here, we define sensitivity to mean understanding how inference about a problem of interest changes with perturbations of the model. In particular it is an example of what we call computational information geometry. The embedding of simple models in much larger information geometric spaces is shown to illuminate these critically important issues.

\section{Chapter 3}

We show how information geometry throws new light on the interplay between goodness-of-fit and estimation, a fundamental issue in statistical inference. A geometric analysis of simple, yet representative, models involving the same population parameter compellingly establishes the main theme of the paper: namely, that goodness-of-fit is necessary but not sufficient for model selection. Visual examples vividly communicate this. Specifically, for a given estimation problem, we define a class of least-informative models, linking these to both nonparametric and maximum entropy methods. Any other model is then seen to involve an informative rotation, often embodying extra-data considerations. We also look at the way that translation of models generates a form of bias-variance trade-off. Overall, our approach is a global extension of pioneering local work by Copas and Eguchi which, we note, was also geometrically inspired.

\section{Chapter 4}

We discuss an approach called spontaneous data learning (SDL) to open novel explanatory paradigm connecting parametrics with nonparametrics. The statistical performance for SDL is explored from information geometric viewpoint, so that SDL gives a new perspective beyond the discussion for robustness or misspecification of parametric model. If the true distribution is exactly in the parametric model, the theory of statistical estimation has been well established, in which any minimum divergence estimator satisfies parametric consistency. We focus on a collapse of the parametric theory perturbing toward a nonparametric setting, where the true distribution may range from unimodality to multimodality; various estimators are targeted and investigated in a class of minimum divergence. In this context a selection of estimators is explored rather than model selection. Specifically we choose the power divergence class under a normal mean model, where the true distribution is, for example, a mixture of $K$ distributions. Then we observe that the local minima of the empirical loss function for the power divergence properly suggest the $K$ means if they are mutually separated in the mixture distribution, and the order of power is appropriated selected. The resulting method for clustering analysis is shown to spontaneously detects the number $K$ of clusters. Further, we observe that the normalized empirical loss function converges to the true density 
function if the power parameter goes to infinity. As a result the power parameter combines between the parametric and nonparametric consistency.

\section{Chapter 5}

We define the notion of the extrinsic Itô projection of a stochastic differential equation (SDE) on a submanifold. This allows one to systematically develop low dimensional approximations to high dimensional SDEs in a differential geometric setting. We consider the example of approximating the non-linear filtering problem with a Gaussian distribution and show how the Itô projection leads to improved approximations in the Gaussian family. We briefly discuss the approximations for more general families of distribution. We perform a numerical comparison of our projection filters with the classical Extended Kalman Filter to demonstrate the efficacy of the approach.

\section{Chapter 6}

Matrix data sets are common nowadays like in biomedical imaging where the Diffusion Tensor Magnetic Resonance Imaging (DT-MRI) modality produces data sets of 3D symmetric positive definite matrices anchored at voxel positions capturing the anisotropic diffusion properties of water molecules in biological tissues. The space of symmetric matrices can be partially ordered using the Löwner ordering, and computing extremal matrices dominating a given set of matrices is a basic primitive used in matrix-valued signal processing. In this letter, we design a fast and easy-to-implement iterative algorithm to approximate arbitrarily finely these extremal matrices. Finally, we discuss on extensions to matrix clustering.

\section{Chapter 7}

Stochastic textures with features spanning many length scales arise in a range of contexts in physical and natural sciences, from nanostructures like synthetic bone to ocean wave height distributions and cosmic phenomena like inter-galactic cluster void distributions. Here we used a data set of 35 surface topographies, each of $2400 \times 2400$ pixels with spatial resolution between 4 and $7 \mu \mathrm{m}$ per pixel, and fitted trivariate Gaussian distributions to represent their spatial structures. For these we computed pairwise information metric distances using the Fisher-Rao metric. Then dimensionality reduction was used to reveal the groupings among subsets of samples in an easily comprehended graphic in 3-space. The samples here came from the papermaking industry but such a reduction of large frequently noisy spatial data sets is useful in a range of materials and contexts at all scales.

\section{Chapter 8}

This artilce summarizes our work on the clustering of financial time series. It was written for a workshop on information geometry and its application for image and signal processing. This workshop brought several experts in pure and applied mathematics together with applied researchers from medical imaging, radar signal processing and finance. The authors belong to the latter group. This document was written as a long introduction to further development of geometric tools in financial applications such as risk or portfolio analysis. Indeed, risk and portfolio analysis 
essentially rely on covariance matrices. Besides that the Gaussian assumption is known to be inaccurate, covariance matrices are difficult to estimate from empirical data. To filter noise from the empirical estimate, Mantegna proposed using hierarchical clustering. In this work, we first show that this procedure is statistically consistent. Then, we propose to use clustering with a much broader application than the filtering of empirical covariance matrices from the estimated correlation coefficients. To be able to do that, we need to obtain distances between the financial time series that incorporate all the available information in these cross-dependent random processes.

\section{Chapter 9}

We consider the geometry and model order specification of a class of density models where the square-root of the distribution is expanded in an orthogonal series. The simplicity of the resulting spherical geometry makes this framework ideal for many applications that rely on information geometric concepts like distances and manifold statistics. Specifically, we demonstrate applications of these models in the computer vision field of object recognition and retrieval. We illustrate how invariant shape representations can be used in conjunction with these probabilistic models to yield state-of-the-art classifiers. Moreover, the viability of formulating classification models that take into account shape deformation in an optimal transport context are investigated, yielding insight into the practicalities of working with the parameter space of the densities versus the Wasserstein measure space approach. The free parameters associated with these square-root estimators can be rigorously selected using the Minimum Description Length (MDL) criterion for model selection. Under these models, it is shown that the MDL has a closed-form representation, atypical for most applications of MDL in density estimation. Experimental evaluation of our techniques are conducted on one, two, and three dimensional density estimation problems in shape analysis, with comparative analysis demonstrating our approach to be state-of-the-art in object recognition and model selection.

\section{Chapter 10}

We propose a dimensionality reduction method for infinite - dimensional measurevalued evolution equations such as the Fokker-Planck partial differential equation or the Kushner-Stratonovich resp. Duncan-Mortensen-Zakai stochastic partial differential equations of nonlinear filtering, with potential applications to signal processing, quantitative finance, heat flows and quantum theory among many other areas. Our method is based on the projection coming from a duality argument built in the exponential statistical manifold structure developed by G. Pistone and co-authors. The choice of the finite dimensional manifold on which one should project the infinite dimensional equation is crucial, and we propose finite dimensional exponential and mixture families. This same problem had been studied, especially in the context of nonlinear filtering, by D. Brigo and co-authors but the $L^{2}$ structure on the space of square roots of densities or of densities themselves was used, without taking an infinite dimensional manifold environment space for the equation to be projected. 
Here we re-examine such works from the exponential statistical manifold point of view, which allows for a deeper geometric understanding of the manifold structures at play. We also show that the projection in the exponential manifold structure is consistent with the Fisher Rao metric and, in case of finite dimensional exponential families, with the assumed density approximation. Further, we show that if the sufficient statistics of the finite dimensional exponential family are chosen among the eigenfunctions of the backward diffusion operator then the statistical-manifold or Fisher-Rao projection provides the maximum likelihood estimator for the Fokker Planck equation solution. We finally try to clarify how the finite dimensional and infinite dimensional terminology for exponential and mixture spaces are related.

\section{Chapter 11}

This paper addresses the problem of learning online finite statistical mixtures of regular exponential families. We first start by reviewing concisely the gradient-based and stochastic gradient-based optimization methods and their generalizations. We then focuses on two stochastic versions of the celebrated Expectation-Maximization (EM) algorithm: Titterington's second-order stochastic gradient EM and Cappé and Moulines' online EM. Depending on which step of EM is approximated, the possible constraints on the mixture parameters may be violated. A justification of these approaches as well as ready-to-use formulas for mixtures of regular exponential families are provided. Finally, to illustrate our study, some experimental comparisons on univariate normal mixtures are provided. 